\title{
Effect of DFT-functional on the energy and electronic characteristics of carbon compounds with the unconventional geometry of the framework
}

\author{
N. V. Novikov ${ }^{1}$, M. M. Maslov ${ }^{1,2}$, K.P. Katin ${ }^{1,2, \dagger}$, V.S. Prudkovskiy ${ }^{2,3}$ \\ †KPKatin@yandex.ru \\ ${ }^{1}$ National Research Nuclear University “MEPhI”, Kashirskoe Shosse 31, Moscow, 115409, Russia \\ ${ }^{2}$ Research Institute for the Development of Scientific and Educational Potential of Youth, \\ Aviatorov St. 14/55, Moscow, 119620, Russia \\ ${ }^{3}$ University of Crete, 71003, Heraklion, Crete, Greece
}

We report how strongly the energy and electronic properties of carbon nanostructures with the unconventional framework calculated by means of density functional theory (DFT) depend on the choice of DFT-functional on the example of carbon $[n, 5]$ prismanes. For comparative analysis we used such characteristics of molecular system as the values of binding energy and energy difference between the highest occupied molecular orbital and the lowest unoccupied molecular orbital (HOMOLUMO gap). We obtained the binding energies and HOMO-LUMO gaps using seven different functionals B3LYP, X3LYP, M11, B3PW91, PBE0, PW, PBE, which belong to generalized gradient approximation and hybrid functionals, and standard 6-31G(d) electronic basis set. We show that the binding energies obtained for the long $[n, 5]$ prismanes with the efficient length of $\sim 150 \AA$ 象ithin the various DFT-functionals can differ by a factor of 1.1, and corresponding HOMO-LUMO gaps can differ by a factor of 23. Additional precision calculations at $\operatorname{CCSD}(\mathrm{T})$ level of theory of $[2,5]$ prismane allow us to conclude that for determining the binding energies of carbon nanostructures with the unconventional framework, the hybrid functionals B3LYP, X3LYP, and M11 are the best choice, whereas for the HOMO-LUMO gap estimation M11 gives the closest to CCSD(T) result. The reported study is of methodological interest for carrying out the correct DFT-calculations with respect to novel nanomaterials with unconventional carbon framework such as hypercubane system, fullerene composites or pillared graphene.

Keywords: density functional theory, polyprismanes, energy and electronic characteristics.

\section{Introduction}

All modern methods of electronic structure calculation can be conventionally divided into three categories: $a b$ initio techniques, semi-empirical schemes and empirical approaches. Density functional theory (DFT) is traditionally considered to be $a b$ initio methods. Although DFT is computationally expensive, it is rather accurate. So, DFT is a very useful tool employed in studies of various physicochemical characteristics of different types of materials and their possible technological applications. DFT is based on the Schrödinger equation that describes the many-body problem [1]. Numerical solving of this problem is a rather complicated task. Therefore, the exchange-correlation functional describing the many-body electron interactions is approximated for simplification [1]. However, the functional choice is a very responsible stage, because different functionals may describe various characteristics of a substance in different ways, and the results obtained may not be consistent with each other $[2,3]$. This is especially true for the nanostructures with a complex carbon-nitrogen atomic structures [4-9].

In this paper, we calculate ground-state properties of carbon nanostructures with the unconventional geometry of the framework, namely, polyprismanes using different DFTfunctionals. Carbon polyprismanes or $[n, m]$ prismanes can be regarded as stacked layers of dehydrogenated cycloalkane molecules, where $m$ is the number of vertices of the closed carbon ring and $n$ is the number of layers [10,11]. For large $n$, they represent single-walled carbon nanotube analogs with an extremely small cross-section as a regular polygon. However, unlike carbon nanotubes, polyprismanes have rectangles on their surfaces instead of hexagons. So, the angles between covalent $\mathrm{C}$ - $\mathrm{C}$ bonds are different from the value $109.5^{\circ}$ usual for the $\mathrm{sp}^{3}$-hybridized carbon orbitals. Here we study in detail the family of $[n, 5]$ prismanes (see Fig. 1 ) at zero temperature and pressure.

We are interested in the behavior of the binding energy and the HOMO-LUMO gap with the increasing of $n$, and we analyze how various exchange-correlation functionals affect these characteristics. 


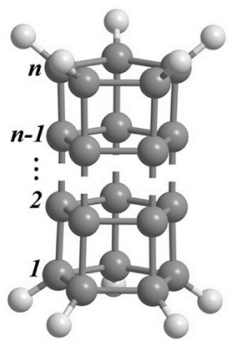

Fig. 1. Atomic structure of carbon [ $n, 5]$ prismanes. Dark balls are the carbon atoms, and light balls are the hydrogen ones.

\section{Computational details}

All DFT-calculations in the presented study were performed using GAMESS program package [12]. The main statement of the density functional theory is that the electronic energy $E$ of the ground state of any quantum system can be found by minimizing some functional depending only on the total electron density $\rho(\mathbf{r})$ of the given system [1]

$$
E[\rho]=T_{s}[\rho]+E_{n e}[\rho]+J[\rho]+E_{X C}[\rho],
$$

where $T_{s}$ is the kinetic energy of a non-interacting system, $E_{n e}$ refers to the interaction between nuclei and electrons, $J$ is the energy of the Coulomb interaction between electrons, and the last term $E_{X C}$ is an exchange-correlation functional, whose exact analytical form is unknown. Various DFT approaches differ from each other only by choosing the form of $E_{X C}$ functional. In this study we choose seven different exchangecorrelation functionals that can be assigned to two broad categories. The first category includes PW [13] and PBE [14] functionals. These functionals refer to the generalized gradient approximation (GGA) [13]. It is assumed that at GGA approximation the functional computed for a certain point of space depends on the electron density at this point, but electron spin and the density gradient (and higher order gradients) are also taken into account

$$
E_{X C}^{G G A}\left[\rho_{\uparrow}, \rho_{\downarrow}\right]=\int \varepsilon_{X C}\left(\rho_{\uparrow}, \rho_{\downarrow}, \nabla \rho_{\uparrow}, \nabla \rho_{\downarrow}, \ldots\right) \rho(\mathbf{r}) \mathrm{d} \mathbf{r} .
$$

Despite the GGA functionals do not contain any adjustable parameters, they possess acceptable accuracy for a wide range of problems, and are computationally efficient. The second category includes B3LYP [15, 16], X3LYP [17], M11 [18], B3PW91 [16], and PBE0 [19] functionals. These functionals are so-called hybrid exchange-correlation functionals, and they are usually constructed as a linear combination of the Hartree-Fock exact exchange functional $E_{X}^{H F}$ and any number of exchange $E_{X}^{D F}$ and correlation $E_{C}^{D F}$ explicit density functionals

$$
E_{X C}^{H Y B}=a E_{X}^{H F}+\sum_{i} b_{i}\left(E_{X}^{D F}\right)_{i}+\sum_{j} c_{j}\left(E_{C}^{D F}\right)_{j} .
$$

The parameters determining the weight of each individual functional are typically specified by fitting the functional predictions to experimental or accurately calculated thermochemical data. For example, one of the most popular hybrid PBE0 functional mixes the PBE exchange energy and Hartree-Fock exchange energy in a set 3 to 1 ratio, along with the full PBE correlation energy

$$
E_{X C}^{P B E 0}=1 / 4 E_{X}^{H F}+3 / 4 E_{X}^{P B E}+E_{C}^{P B E},
$$

where $E_{X}^{H F}$ is the Hartree-Fock exact exchange functional, $E_{X}^{P B E}$ is the PBE exchange functional, and $E_{C}^{P B E}$ is the PBE correlation functional. Note that for all functionals used, the calculations were carried out using the standard Pople 6-31G(d) electronic basis set [20,21].

\section{Results and discussion}

First of all, we performed structural optimization of [n,5]prismane family with $n=2 \div 10$ using all above mentioned DFT-functionals. After that, binding energies and HOMO-LUMO gaps were obtained. The binding energies of $[n, 5]$ prismanes $\mathrm{C}_{l} \mathrm{H}_{k}$ are calculated by the equation

$$
E_{b}=1 / N_{a t}\left[k E(\mathrm{H})+l E(\mathrm{C})-E_{t o t}(\mathrm{PP})\right],
$$

where $N_{a t}=k+l$ is the total number of atoms in the polyprismane, $E(\mathrm{C})$ and $E(\mathrm{H})$ are the energies of isolated carbon and hydrogen atoms, respectively, and $E_{\text {tot }}(\mathrm{PP})$ is the total energy of the corresponding polyprismane. The polyprismane with higher binding energy $E_{b}$ (lower potential energy) is more thermodynamically stable and vice versa. HOMO-LUMO gap $\Delta_{\mathrm{HL}}$ is defined as the energy gap between the highest occupied molecular orbital and the lowest unoccupied molecular orbital. The binding energies and the HOMO-LUMO gaps obtained for some $[n, 5]$ prismanes are summarized in Tables 1 and 2, respectively. From the Figs. 2 and 3, one can see that the DFT-functional choice significantly affects the values of binding energies and HOMO-LUMO gaps.

For a better understanding of the differences in values of binding energies and HOMO-LUMO gaps obtained using various DFT-functionals, we approximate the dependencies $E_{b}(n)$ and $\Delta_{\mathrm{HL}}(n)$ for the case of large $n \rightarrow \infty$. Approximations
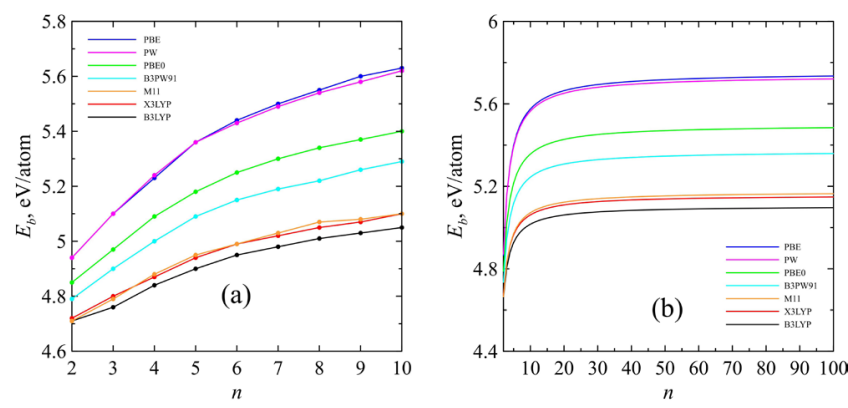

Fig. 2. Binding energies $E_{b}$ versus the number of $\mathrm{C}_{5}$-rings $n$ obtained in the frame of density functional theory using various functionals: direct calculations (a) and further approximation (b).
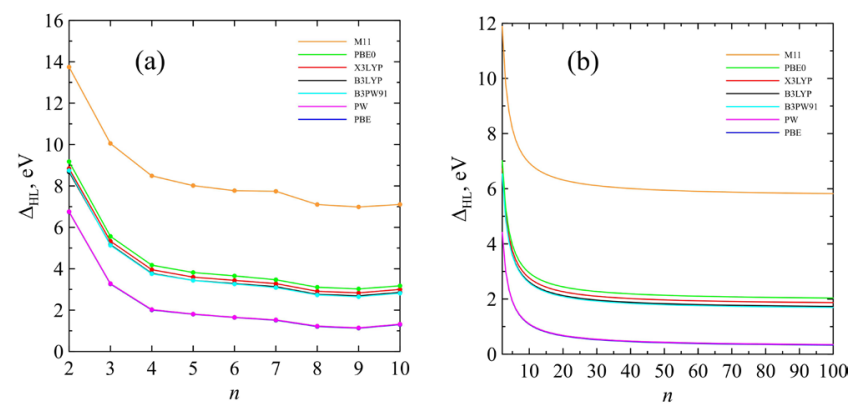

Fig. 3. HOMO-LUMO gaps $\Delta_{\mathrm{HL}}$ versus the number of $\mathrm{C}_{5}$-rings $n$ obtained in the frame of density functional theory using various functionals: direct calculations (a) and further approximation (b). 
Table 1. Binding energies (eV/atom) of [ $n, 5]$ prismanes obtained using different DFT-functionals and 6-31G(d) electronic basis set.

\begin{tabular}{|c|c|c|c|c|c|c|c|}
\hline Functional & \multirow{2}{*}{ PW } & PBE & PBE0 & \multirow{2}{*}{ B3LYP } & \multirow{2}{*}{ X3LYP } & \multirow{2}{*}{ B3PW91 } & M11 \\
\hline$n$ & & & & & & & \\
\hline 2 & 4.94 & 4.94 & 4.85 & 4.71 & 4.72 & 4.79 & 4.71 \\
\hline 3 & 5.10 & 5.10 & 4.97 & 4.76 & 4.80 & 4.90 & 4.79 \\
\hline 4 & 5.24 & 5.23 & 5.09 & 4.84 & 4.87 & 5.00 & 4.88 \\
\hline 5 & 5.36 & 5.36 & 5.18 & 4.90 & 4.94 & 5.09 & 4.95 \\
\hline 6 & 5.43 & 5.44 & 5.25 & 4.95 & 4.99 & 5.15 & 4.99 \\
\hline 7 & 5.49 & 5.50 & 5.30 & 4.98 & 5.02 & 5.19 & 5.03 \\
\hline 8 & 5.54 & 5.55 & 5.34 & 5.01 & 5.05 & 5.22 & 5.07 \\
\hline 9 & 5.58 & 5.60 & 5.37 & 5.03 & 5.07 & 5.26 & 5.08 \\
\hline 10 & 5.62 & 5.63 & 5.40 & 5.05 & 5.10 & 5.29 & 5.10 \\
\hline
\end{tabular}

Table 2. HOMO-LUMO gaps (eV) of [n,5]prismanes obtained using different DFT-functionals and 6-31G(d) electronic basis set.

\begin{tabular}{|c|c|c|c|c|c|c|c|}
\hline Functional & \multirow[t]{2}{*}{ PW } & \multirow[t]{2}{*}{ PBE } & \multirow[t]{2}{*}{ PBE0 } & \multirow[t]{2}{*}{ B3LYP } & \multirow[t]{2}{*}{ X3LYP } & \multirow[t]{2}{*}{ B3PW91 } & \multirow[t]{2}{*}{ M11 } \\
\hline$n$ & & & & & & & \\
\hline 2 & 6.76 & 6.75 & 9.18 & 8.68 & 8.84 & 8.74 & 13.75 \\
\hline 3 & 3.28 & 3.27 & 5.57 & 5.17 & 5.33 & 5.14 & 10.05 \\
\hline 4 & 2.03 & 2.01 & 4.17 & 3.78 & 3.95 & 3.76 & 8.49 \\
\hline 5 & 1.81 & 1.80 & 3.82 & 3.44 & 3.60 & 3.43 & 8.02 \\
\hline 6 & 1.65 & 1.65 & 3.65 & 3.28 & 3.44 & 3.26 & 7.77 \\
\hline 7 & 1.53 & 1.51 & 3.47 & 3.13 & 3.28 & 3.09 & 7.74 \\
\hline 8 & 1.23 & 1.21 & 3.11 & 2.76 & 2.91 & 2.73 & 7.10 \\
\hline 9 & 1.14 & 1.13 & 3.03 & 2.69 & 2.83 & 2.65 & 6.99 \\
\hline 10 & 1.32 & 1.30 & 3.17 & 2.86 & 3.00 & 2.82 & 7.11 \\
\hline
\end{tabular}

Table 3. Coefficients $A$ and $B$ in the $E_{b}(n)=A-B n^{-1}$ dependence for the different DFT-functionals.

\begin{tabular}{|c|c|c|c|c|c|c|c|}
\hline Functional & \multirow{2}{*}{ PW } & PBE & PBE0 & B3LYP & X3LYP & \multirow{2}{*}{ B3PW91 } & M11 \\
\cline { 1 - 7 } Coefficient & & & & & & & \\
\hline$A$ & 5.738 & 5.753 & 5.499 & 5.106 & 5.158 & 5.372 & 5.174 \\
\hline$B$ & 1.734 & 1.775 & 1.419 & 0.892 & 0.966 & 1.273 & 1.020 \\
\hline
\end{tabular}

Table 4. Coefficients $A$ and $B$ in the $\Delta_{\mathrm{HL}}(n)=A+B n^{-1}$ dependence for the different DFT-functionals.

\begin{tabular}{|c|c|c|c|c|c|c|c|}
\hline Functional & \multirow{2}{*}{ PW } & \multirow{2}{*}{ PBE } & PBE0 & B3LYP & X3LYP & \multirow{2}{*}{ B3PW91 } & M11 \\
\cline { 1 - 6 } Coefficient & & & & & & & \\
\hline$A$ & 0.265 & 0.248 & 1.932 & 1.633 & 1.769 & 1.593 & 5.698 \\
\hline$B$ & 8.308 & 8.327 & 10.169 & 9.829 & 9.931 & 9.893 & 12.376 \\
\hline
\end{tabular}

were made using SciDAVis program package [22]. The corresponding analytical forms (see Figs. $2 \mathrm{~b}$ and $3 \mathrm{~b}$, respectively) are

$$
E_{b}(n)=A-B n^{-1}, \quad \Delta_{\mathrm{HL}}(n)=A+B n^{-1} .
$$

The $A$ and $B$ coefficients are presented in Tables 3 and 4 for every DFT-functional used. From the Figs. 2 and 3, one can see that the minimum value of binding energy corresponds to the B3LYP functional, and the maximum one corresponds to the PBE functional, whereas the minimum value of HOMO-LUMO gap corresponds to the PBE functional, and the maximum one corresponds to M11 functional. It can be estimated that

$$
\left.\frac{E_{b}^{\mathrm{PBE}}}{E_{b}^{\mathrm{B} 3 \mathrm{LY}}}\right|_{n \rightarrow \infty} \approx 1.1,\left.\frac{\Delta_{\mathrm{HL}}^{\mathrm{M} 11}}{\Delta_{\mathrm{HL}}^{\mathrm{PBE}}}\right|_{n \rightarrow \infty} \approx 23 .
$$

Thus, the choice of DFT-functional especially in the case of electronic properties calculations should be made carefully.

To formulate the recommendations for the using of specific DFT-functional in the case of carbon systems with the unconventional geometry of the framework, we made additional precision calculations at $\operatorname{CCSD}(\mathrm{T})$ level of theory [23] of the smallest [2,5]prismane. The same basis set $6-31 G(d)$ is used for coupled-clusters calculations. At this level of theory the binding energy and HOMO-LUMO gap of [2,5]prismane are equal $4.54 \mathrm{eV} /$ atom and $15.9 \mathrm{eV}$, 
respectively. Thus, for the binding energies calculation of carbon nanostructures with the unconventional framework, the hybrid functionals B3LYP, X3LYP, and M11 give the closest to $\operatorname{CCSD}(\mathrm{T})$ results, whereas for the HOMO-LUMO gap estimation M11 is a good choice. Unfortunately, data obtained by PW and PBE functionals poorly matched with the $\operatorname{CCSD}(\mathrm{T})$ results. So, although GGA functionals are successfully used for calculation of various physicochemical properties of crystals, they are poorly suited for describing the energy and electronic characteristics of isolated molecules with a non-traditional structure of the carbon framework.

\section{Conclusions}

In this paper, we have reported the values of binding energies and HOMO-LUMO gaps predicted employing seven various DFT-functionals for carbon $[n, 5]$ prismanes with $n=2 \div 10$. It is shown that the binding energies and HOMO-LUMO gaps obtained for $[n, 5]$ prismanes can significantly vary depending on the chosen functional. One can see that B3LYP and PBE functionals yield results for binding energies at two extreme ends, whereas the same statement is true for M11 and PBE functionals in the case of HOMO-LUMO gaps calculation. Data comparison with the results obtained within the more strict coupled-clusters theory shows that the hybrid functionals are better suited for describing the energy and electronic characteristics of isolated molecules with a non-traditional structure of the carbon framework than the GGA functionals.

Acknowledgments. The reported study was funded by RFBR, according to the research project No.16-32-60081 mol_a_dk.

\section{References}

1. R. G. Parr, W. Yang. Density-Functional Theory of Atoms and Molecules. NY: Oxford University Press (1990) 333 p.

2. G. S. Manyali. IOSR Journal of Applied Physics 8, 54-58 (2016), DOI: 10.9790/4861-08135458

3. G. Zhang, C.B. Musgrave. J. Phys. Chem. A 111, 1554 - 1561 (2007), DOI: 10.1021/jp061633o

4. E.A. Belenkov, M.M. Brzhezinskaya, V.A. Greshnyakov. Nanosystems: Physics, Chemistry, Mathematics 8, 127 136 (2017), DOI: 10.17586/2220-8054-2017-8-1-127-136

5. E.A. Belenkov, V.A. Greshnyakov. J. Mater. Sci. 50,
7627 - 7635 (2015), DOI: 10.1007/s10853-015-9325-1

6. K.A. Krylova, Yu.A. Baimova, S.V. Dmitriev, R. R. Mulyukov. Phys. Solid State 58, 394-401 (2016), DOI: $10.1134 /$ S1063783416020189

7. R. A. Brazhe, A. I. Kochaev, V. S. Nefedov. Phys. Solid State 54, 1430 - 1432 (2012), DOI: 10.1134/S1063783412070098

8. M. M. Maslov, K. P. Katin, A. I. Avkhadieva, A. I. Podlivaev. Russ. J. Phys. Chem. B 8, 152 - 157 (2014), DOI: 10.1134/ S1990793114020195

9. K.P. Katin, M. M. Maslov. J. Phys. Chem. Solids 108, 82 - 87 (2017), DOI: 10.1016/j.jpcs.2017.04.020

10. R.M. Minyaev, V.I. Minkin, T.N. Gribanova, A. G. Starikov, R. Hoffmann. J. Org. Chem. 68, 8588- 8594 (2003), DOI: 10.1021/jo0349101

11. K.P. Katin, S.A. Shostachenko, A.I. Avkhadieva, M. M. Maslov. Adv. Phys. Chem. 2015, 506894 (2015), DOI: $10.1155 / 2015 / 506894$

12. M. W. Schmidt, K. K. Baldridge, J. A. Boatz, S. T. Elbert, M.S. Gordon, J.H. Jensen, S. Koseki, N. Matsunaga, K.A. Nguyen, S. Su, T.L. Windus, M. Dupuis, J. A. Montgomery. J. Comp. Chem. 14, 1347 - 1363 (1993), DOI: $10.1002 /$ jcc.540141112

13. J.P. Perdew, J.A. Chevray, S.H. Vosko, K.A. Jackson, M. R. Pederson, D. J. Singh, C. Fiolhais. Phys. Rev. B 46, 6671 - 6687 (1992), DOI: 10.1103/PhysRevB.46.6671

14. J.P. Perdew, K. Burke, M. Ernzerhof. Phys. Rev. Lett. 77, 3865 - 3868 (1996), DOI: 10.1103/PhysRevLett.77.3865

15. C. Lee, W. Yang, R. G. Parr. Phys. Rev. B 37, 785 (1988), DOI: 10.1103/PhysRevB.37.785

16. A.D. Becke. J. Chem. Phys. 98, 5648 (1993), DOI: $10.1063 / 1.464913$

17. X. Xu, Q. Zhang, R.P. Muller, W.A. Goddard. J. Chem. Phys. 122, 014105 (2005), DOI: 10.1063/1.1812257

18. R. Peverati, D. G. Truhlar. J. Phys. Chem. Lett. 2, 2810 - 2817 (2011), DOI: 10.1021/jz201170d

19. C. Adamo, V. Barone. J. Chem. Phys. 110, 6158-6170 (1999), DOI: 10.1063/1.478522

20. R. Ditchfield, W. J. Hehre, J. A. Pople. J. Chem. Phys. 54, 724 (1971), DOI: 10.1063/1.1674902

21. R. Krishnan, J. S. Binkley, R. Seeger, J. A. Pople, J. Chem. Phys. 72, 650 (1980), DOI: 10.1063/1.438955

22. SciDAVis - Scientific Data Analysis and Visualization: scidavis.sourceforge.net

23. T. Helgaker, P. Jorgensen, J. Olsen. Molecular ElectronicStructure Theory. Wiley: New York (2000) P. 793-796. 